\title{
LA BIOPIRATERÍA DE LOS RECURSOS DE LA MEDICINA INDÍGENA TRADICIONAL EN EL ESTADO CHIAPAS, MÉXICO -EI caso ICBG-MAYA-
}

\author{
Roberto Rafael Alarcón Lavín \\ omiech@ prodigy.net.mx \\ Comisión para el Desarrollo de los Pueblos Indígenas- \\ Organización de Médicos Indígenas del Estado de Chiapas \\ México
}

\section{RESUMEN}

Este artículo pretende un breve análisis de la biopiratería experimentada y resistida por parte de los pueblos mayas de Chiapas. Para ello se da cuenta de acuerdos y normas internacionales para la protección de los recursos y conocimientos de los pueblos indígenas; las bases económicas y características, en los ámbitos mundial y nacional, que dan la pauta para la nueva ola de proyectos de bioprospección de los recursos vegetales de la medicina indígena tradicional, dirigida por los países del norte hacia los del sur; algunas experiencias de su implantación en Sudamérica y otros países subdesarrollados; se explican los elementos más importantes del proyecto de bioprospección denominado «Investigación farmacéutica y uso sustentable del conocimiento etnobotánico y biodiversidad en la Región Maya de Los Altos de Chiapas, México», que fue operado por parte de El Colegio de la Frontera Sur, Ecosur, la Universidad de Georgia — con apoyo económico del gobierno de Estados Unidos - y la compañía biotecnológica molecular Nature Limited de Gales, Reino Unido; por último, se describe la lucha que llevó a cabo el Consejo de Médicos y Parteras Indígenas Tradicionales de Chiapas, Compitch, para que fuera cancelado el mencionado proyecto, así como algunas propuestas alternativas de Compitch a este proyecto de biopiratería.

Palabras clave: bioprospección-biopiratería, ICBG-Maya, Compitch. 
ABSTRACT

With this paper i pretend to bring a brief analysis on how biopiracy has been experienced and manage by the Mayas of Chiapas. For this we have to mention agreements and international norms for the protection of the resources and knowledge of original people; economical background and its characteristics in the international and national level, which give the pattern for the new wave of bioprospective projects on vegetal resources used in indigenous medicine, directed from de north to the south countries; some experiences of it in South America and other undeveloped countries: the explanation of most relevant elements of the project ICBG-Maya, operated by El Colegio de la Frontera Sur, Ecosur, Georgia University - with the support of the government of the United States - and biotechnology laboratory Molecular Nature Limited form Whales, UK; in the last term we describe the struggle that the Consejo de Médicos y Parteras Indígenas Tradicionales de Chiapas, Compitch, to evict the project and some alternative proposals.

Key words: bioprospection-biopiracy, ICBG-Maya, Compitch. 


\section{INTRODUCCIÓN}

Desde tiempos prehispánicos en Mesoamérica la herbolaria medicinal y su utilización tanto en humanos como en animales fue una actividad ejercida e influenciada con elementos socioculturales hasta cierto punto fáciles de entender: intercambio o trueque individual de plantas medicinales; aprendizaje de su utilización por trasmisión oral, por imitación de conductas animales o a través de sueños; experimentación empírica de su efectividad sobre procesos mórbidos; clasificación taxonómica con base en características etnobotánicas simplificadas; recolección en ambientes silvestres para su utilización individual o familiar.

Con la invasión española nuevos elementos, o la redirección de algunos ya existentes, se adhirieron a la herbolaria medicinal: plantas introducidas por los españoles; intercambio de plantas con esclavos negros; incidencia de primordios de la investigación bioquímica para determinar sus propiedades terapéuticas; exploraciones botánicas extensas en busca de plantas con posibilidades terapéuticas factibles de reproducir masiva y comercialmente; prohibición de su comercialización a otros países por parte del país invasor; inicio del secreto comercial. Así pues, con estos elementos, las plantas medicinales y su uso fueron rodeándose de un halo complicado, tan complicado que provocó su fetichización, convirtiéndose en una mercancía factible de detonar procesos de acumulación de capital. Con implementos técnicos e instrumentos ideológicos, con nombres diversos pero con las mismas intenciones de explotar los recursos naturales para acumular capital, estos «nuevos» procesos se han llevado a cabo constantemente a través de la historia en el marco de las relaciones asimétricas entre los pueblos dominantes y los dominados: las plantas medicinales fetichizadas y convertidas en mercancía.

En la actualidad, en este proceso de acumulación de capital de las empresas farmacéuticas nacionales y trasnacionales, que utilizan plantas medicinales para producir sus fitofármacos ubicamos, entrelazadas, una larga cadena-red de procesos donde inciden las ciencias sociales y biológicas, como en: las disposiciones reglamentarias jurídicas nacionales e internacionales - Convenio de Biodiversidad Biológica; Derechos de patente; Derechos de 
Propiedad Intelectual; legislaciones nacionales sobre biodiversidad, etc.-—; los estudios etnobotánicos — sustracción de información empírica sobre la utilización de las plantas medicinales a los integrantes de las culturas indígenas originarias: formularia indígena-; los estudios médicos de los perfiles patológicos y de mortalidad de los países en los cuales se comercializarán los fitofármacos producidos; los estudios de las características botánicas de las plantas seleccionadas para combatir las patologías encontradas en los perfiles de morbilidad y mortalidad de los países con amplio poder de compra de fitofármacos; los estudios georeferenciales para la elaboración de archivos florísticos de herbolaria medicinal de las regiones indígenas, con propósitos de utilización terapéutica y de comercialización; los estudios farmacológicos en animales de laboratorio, aplicándoles la formularia indígena recopilada y seleccionada - generalmente por biólogos, antropólogos o etnobiólogospara incidir en perfiles patológicos y de mortalidad de los países en donde se comercializarán los fitofármacos; los estudios fitoquímicos de las plantas, en animales y grupos humanos seleccionados.

En 2010, un frasco de un producto medicinal de origen herbolario, además de su nombre químico rebuscado y casi incomprensible para el lego en materia fitobiomédica, contiene todos los elementos de este proceso de fetichización sociohistórico.

En este ensayo, trataremos de incursionar en la fetichización de algunos de estos elementos para «desfetichizarlos»y, con ello, explicarnos, en parte, por qué no hemos avanzado en el uso de la herbolaria nacional para beneficio de todos los enfermos pobres del mundo.

\section{REGLAMENTACIÓN MUNDIAL SOBRE LA PROTECCIÓN DE LOS CONOCIMIENTOS Y RECURSOS DE LAS POBLACIONES LOCALES}

El Convenio sobre Diversidad Biológica, CDB, firmado en 1992 durante la Conferencia sobre Medio Ambiente y Desarrollo, debiera ser la base legal para la distribución de beneficios que resulten del uso de la diversidad biológica y de su conocimiento asociado. Desgraciadamente, por un lado, algunos de sus artículos, que parecieran favorecer a los 
pueblos indios y, por el otro, la ambigüedad de otros (Chadler 1994: 1, 6) han despertado suspicacias tanto en los propios pueblos indios, como en el gobierno de Estados Unidos de Norteamérica, EEUU, dificultando su aplicación práctica. La interpretación del CDB, por múltiples consultores, ${ }^{1}$ no ha desembocado en alternativas de solución viables para la protección de los conocimientos y recursos de las poblaciones locales.

No obstante de estas ambigüedades, CDB contiene apartados en los cuales se explicita la soberanía de los Estados sobre sus recursos biológicos. ${ }^{2}$ Esta situación ha ocasionado que EEUU - líder mundial en biotecnología - no lo haya ratificado. Al no ser acatados estos acuerdos la distribución de beneficios para las poblaciones que poseen los recursos biológicos y el conocimiento sobre ellos no se cumple. Las empresas asentadas en este país, que pudieran ser de cualquier nacionalidad, no tienen el mandato de cumplir con la letra y espíritu de CDB. Así pues, empresas y laboratorios prefieren asentar sus filiales en EEUU para evitar la letra y espíritu de CDB. Por ejemplo, Manfred Schneider, director de la farmacéutica Bayer A. G., expresa, de manera bastante diplomática, las razones para esta reubicación: «Norteamérica, EEUU, no ha reemplazado a Alemania como sitio de negocios, pero hay ciertas actividades innovadoras que se llevan a cabo de mejor manera en los EEUU» (Nash 1995: D1, 5).

Los conocimientos, sobre los usos de los recursos naturales, que las poblaciones locales de los países del hemisferio sur poseen están siendo conducidos a incorporarse en la lógica económica de la Propiedad Intelectual, PI, y los Derechos que la regulan, DPI. Se basa esta lógica en los derechos individuales de «poseer algo» —en este caso un conocimiento sobre el uso de un recurso natural- para comercializarlo; y su protección, para que otro(s) individuo(s) no obtenga(n) ganancias monetarias a partir de este conocimiento de la utilización del recurso natural. Por ejemplo, el costo de lanzar una nueva medicina es tan extraordinariamente alto que, inclusive, es complicado asignar costos dentro de una empresa farmacéutica dada. Los portavoces de las industrias presentan cifras exorbitantes; el presidente de Pharmaceutical Research and Manufactures of América, en una carta al 
editor de la revista The Economist, escribe lo siguiente: «Según un estudio de 1996 del Boston Consulting Group, cuesta en promedio 500 millones de dólares desarrollar una sola medicina» (Mossinghoff 1996: 8); mientras que el asesor de patentes del laboratorio Merck \& Co. reporta un número significante menor: «La estimación actual de estas empresas de alto riesgo en un promedio de 12 años, desde el descubrimiento hasta el mercado, es de más de 350 millones de dólares» (Tribble 1995: 97-104).

Sea el costo 350 o 500,000,000 de dólares, lo que cuenta en el fondo es que, una vez que una medicina es puesta en el mercado, los empresarios de países que no otorgan patentes sobre medicinas pueden encontrar la patente por el servidor de patentes de IBM, http://www.patents.ibm.com, que provee acceso a más de 26 años de descripciones de patentes e imágenes de la Oficina de Patentes y Marcas de EEUU -U.S. Patent \& Trademark Office, USP-TO- e intentar manufacturarla en sus países con un gasto que es una fracción de los costos fijos de investigación y desarrollo originales, es decir, mucho menos de las cifras anteriormente expresadas. Esta desigualdad en la protección por patentes entre los países desarrollados y los países en vías de desarrollo produce una asimetría en la investigación y desarrollo de las medicinas. La industria farmacéutica en los países desarrollados y en los países en desarrollo tiene poco incentivo para hacer investigación y desarrollo que pueda volverse conocimiento público. Así, las enfermedades que afligen a decenas de millones de personas de países en vías de desarrollo son ignoradas por el mercado privado.

Esta lógica del mercado mundial individualizado — la patente, por ley, es individual-, contrasta con la lógica cultural de los médicos indígenas tradicionales del Consejo de Organizaciones de Médicos y Parteras Indígenas Tradicionales de Chiapas, Compitch, que en torno a la Tercera Reunión de la Mesa de Negociación Tripartita ${ }^{3}$ declara en el apartado IV: 
Para el Compitch todos los proyectos de bioprospección con fines comerciales o que tienen como propósito adquirir una protección bajo el sistema de patentes y propiedad intelectual, son incompatibles con el marco jurídico nacional que regula las bases de la vida y la cultura asociada a ella, lo que es particularmente claro respecto a la cultura, usos, costumbres, formas de organización colectiva de los pueblos y comunidades indígenas. ${ }^{4}$

\section{LA OLA DE BIOPROSPECCIÓN-BIOPIRATERÍA DE LOS PAÍSES DEL NORTE HACIA LOS DEL SUR}

La biodiversidad se ha convertido en un recurso y un bien. El desarrollo de la biotecnología, en los países desarrollados, como una industria multimillonaria ha dado un valor mercantil incalculable a la biodiversidad porque dicha industria depende de la biodiversidad mundial para la obtención de sus materias primas, a saber, los recursos genéticos.

La bioprospección a menudo se concentra en las tierras indígenas, pues las áreas tropicales donde no ha tenido lugar el desarrollo industrial albergan las más diversas colecciones de recursos genéticos a escala mundial. Aproximadamente $80 \%$ de la población rural de los países en desarrollo utiliza las plantas medicinales para atender sus problemas de salud (Ribeiro 2002: 19). Esta relación armónica y conocimiento de la flora medicinal local han sido trasmitidos de generación en generación por las poblaciones indígenas y campesinas, y su conocimiento se concibe socialmente como un bien eminentemente colectivo. Las plantas medicinales, dicen los j'iloletik maya-tsotsiles, «...las puso Dios en la tierra para que las cuidáramos, para que las usáramos en beneficio de nuestros hermanos, no para que se apropie una persona de ellas» (OMIECH 1989: 72).

La relación que los médicos indígenas tienen en la utilización de los recursos vegetales los ha llevado a diferenciar qué parte de la planta se usa para cada enfermedad, cualidades térmicas de cada planta, cuáles son venenosas, cantidad en su uso, forma y tiempo de cosecha y reproducción; además, de una amplia clasificación botánica nativa, basada en la forma, color, sabor, uso, lugar de crecimiento de cada vegetal. Este vasto conocimiento, 
como explicaremos más adelante, es importantísimo y lucrativo para los proyectos de bioprospección de las empresas multinacionales farmacéuticas, ya que les significa un enorme ahorro en investigación al indicar qué recursos son más útiles y qué camino pueden tomar.

La diversidad de la vida en territorio tradicional de muchos pueblos indígenas es una mina de oro para la industria de la tecnología. Como regla general la biodiversidad en el mundo aumenta desde los polos hacia los trópicos; los máximos valores se encuentran en los bosques lluviosos tropicales y de estos en los lugares más montañosos; lugares en donde habitan la mayoría de las poblaciones originarias de países pobres (Carrera de la Torre 1995: 12). Por ejemplo, una corporación o empresa que busca producir un fertilizante para el suelo podría medir el efecto de diferentes hongos en los suelos, en busca de uno que estimule mejor el crecimiento de determinados cultivos. Se estima que 25,000 a 80,000 especies de plantas superiores se encuentran en territorios del hemisferio sur (Schultez 1990: 9-36). El número de especies de plantas que crecen en el hemisferio norte es menor en comparación con su abundancia natural en los bosques lluviosos tropicales. Esto aumenta considerablemente la probabilidad para la empresa de desarrollar un producto comercializable. Así pues, las corporaciones farmacéuticas en particular dependen de los conocimientos medicinales indígenas para ahorrar tiempo y dinero en la localización e identificación de recursos genéticos con cualidades medicinales para contrarrestar, principalmente, enfermedades de los perfiles de morbi-mortalidad de los países desarrollados — cáncer, enfermedades del sistema nervioso, etcétera.

La mayoría de los proyectos de bioprospección que se han instalado en los países del sur —sobre todo de Estados Unidos $-{ }^{5}$ responden, principalmente, a dos situaciones: primera, la pérdida de liderazgo de Estados Unidos en otras ramas económicas frente a Japón y Alemania y, segunda, la supremacía indiscutible de Estados Unidos en cuanto a biotecnología de punta (Massieu 1997: 70-71). 
De los productos que comercializa la industria farmacéutica de los países desarrollados a escala mundial 60\% provienen de los países del sur (Torres 1996: 13). Según la Fundación Internacional del Progreso Rural - Rural Advancement Foundation International, RAFIde Canadá, se estima que tres cuartas partes de todos los fármacos de prescripción de origen vegetal fueron descubiertos debido al uso previo en la medicina indígena (Ribeiro 2002: 19):

Entre 1950 y 1980, el 25 por ciento de las medicinas de receta vendidas en Estados Unidos tenía su base en fármacos derivados de plantas. En la actualidad, el 40 por ciento de las medicinas que se encuentran en pruebas clínicas son también derivados de plantas. De estas, tres cuartas partes están basadas en plantas que son utilizadas por indígenas, lo que permitió su posterior «descubrimiento» por parte de las empresas farmacéuticas. Se estima que el valor económico total de los fármacos derivados de plantas en Estados Unidos es mayor de 68 millones de dólares anuales (Ribeiro 2002: 19).

En 1993, los Institutos Nacionales de Salud del Gobierno de Estados Unidos destinaron seiscientos millones de dólares para investigación sobre drogas relacionadas con la biodiversidad y con productos médicos del mundo natural (Sierra 1999: 21-22). El mercado mundial anual para dichos fármacos derivados de plantas medicinales se estima, según cifras conservadoras, en ciento sesenta billones de dólares (Sierra 1999: 21-22), de los cuales las comunidades de las que se obtuvo el conocimiento y las plantas no reciben regalías. Según la RAFI, los países no industrializados o «en desarrollo» pierden casi cinco y medio billones de dólares al año de regalías no obtenidas por compañías farmacéuticas y de semillas (McNally 1996: 221-228).

En el ámbito nacional, la merma del presupuesto destinado a centros de investigación tecnológica y universidades ha venido disminuyendo considerablemente. ${ }^{6}$ Esta situación ha provocado que dichas instituciones acepten los recursos económicos y una supuesta trasferencia de tecnología por parte de los países desarrollados, firmando convenios inequitativos para los países huéspedes y, lo más importante, sin la participación plena de 
los directamente afectados en dichos proyectos. Así, tenemos como ejemplos los convenios UNAM-Diversa (Nadal 1999) y Ecosur-Universidad de Georgia-Molecular Nature Limited. ${ }^{7}$ Una de las características principales de estos proyectos es su presentación como proyectos de investigación académica $^{8}$ y no como proyectos de orden estrictamente económico, lo que provoca que algunos investigadores nacionales asociados $-\mathrm{y}$ bien remunerados -9 a estos proyectos los defiendan a ultranza.

\section{EXPERIENCIAS DE BIOPIRATERÍA DE LOS RECURSOS DE LA MEDICINA INDÍGENA EN PAÍSES DEL TERCER MUNDO}

La biopiratería de recursos naturales y el conocimiento de su utilización han estado presentes en nuestros países desde los tiempos inmediatos posteriores a la invasión de América, por parte de los europeos.

La introducción de plantas medicinales a Europa, en cierta forma, produjo una revolución en el arte de curar del viejo mundo. La obra de Monárdes con la descripción de pocas docenas de tales plantas y los resultados obtenidos por él mismo en sus tratamientos en Sevilla, la ciudad más populosa de España en esa época, tuvo gran difusión (Pérez 2007: 1).

El conocimiento herbolario azteca, plasmado en el Libellus de Medicinalibus Indorum Herbis, de Martín de la Cruz, fue enviado a Europa y utilizado por manos de frailes y boticarios (De la Cruz 1991: 3-5).

Tomás López Medel, en su Tratado de los tres elementos: agua, aire y tierra, refiere la siguiente cita:

De manera que las occidentales Indias no solamente nos hacen ricos con sus riquezas quedándose dichas ellas en pobreza pero aún la salud par(a) nuestros cuerpos nos envían envuelta en mil géneros de cosas que (la) naturaleza en ellas cría y a trueque de estos beneficios tantos aún la salud espiritual que tan debidamente les debemos procurar con la predicación de la palabra de Dios para su 
enseñamiento les denegamos, siendo deuda a que muy obligados estamos y derecho que delante de Dios y el mundo nos pueden muy bien pedir (López 1784, f187).

En el año 1903 el Instituto Médico Nacional había colectado más de 17,000 ejemplares botánicos, de los cuales se había realizado el análisis químico de 122 y extraído 700 compuestos químicos (Lozoya 1997: 15-16).

Richard Evans S., en la amazonia colombiana, ha podido estudiar más de 1,500 especies pertenecientes a 600 géneros y 145 familias (Naranjo 1996: 6). Este mismo investigador se pregunta, en su famoso libro Plantas de los dioses sobre si la comprensión del uso y la composición química de estas drogas nos llevarán a descubrir nuevas herramientas farmacéuticas para la experimentación y el tratamiento psiquiátrico (Evans 1993: 9).

Actualmente, las compañías farmacéuticas - y sus intermediarios - realizan prospecciones genéticas en zonas tropicales de manera rutinaria en búsqueda de valiosos compuestos farmacológicos (Sierra 1999: 10-11). «En la mayoría de los casos, según describe el economista ambiental José Carlos Fernández, de El Colegio de la Frontera Sur, Ecosur, de San Cristóbal de Las Casas, las empresas ofrecen a uno o a varios miembros de la comunidad un magro pago único por la explotación de sus conocimientos y la riqueza de sus territorios» (Sierra 1999: 10-11).

Conrad Gorinsky posee la patente para el cunaniol, que muchas comunidades indígenas de la cuenca amazónica utilizan como un veneno en la pesca. Gorinsky tiene los derechos exclusivos de comercialización de cualquier producto derivado de esta planta (Rothschild 1996: 36).

En la India, el árbol de neem ha sido utilizado como pesticida durante cientos de años y es sagrado para los hindúes. WR Grace and Company posee la patente norteamericana gracias a una pequeñísima modificación que dicha compañía hizo del neem. Ningún crédito o 
beneficio han recibido los agricultores tradicionales de la India que se consideran los verdaderos cultivadores de esta planta (Rothschild 1996: 36).

Cuando, por ejemplo, las corporaciones farmacéuticas desarrollan nuevas drogas basadas en el conocimiento indígena de las plantas medicinales, casi siempre los pueblos indígenas no tienen una base legal para competir con ellas. Esto se debe a que las corporaciones cambian el material original de alguna manera o utilizan sólo una parte del recurso, como un compuesto químico aislado (Rothschild 1996: 40).

En 1986, Loren Miller y la International Plant Medicine Corporation recibieron la patente sobre una variedad de la planta conocida científicamente como Banisteripsis caapi, o como Ayahuasca en las comunidades de la amazonia. Los pueblos indígenas de toda la región amazónica se han mostrado sumamente molestos por la patente obtenida por este biopirata de una planta que es sagrada para ellos. La ambición comercial de Miller lo ha llevado a realizar la siguiente propuesta que presentamos textualmente del libro del Centro por los Derechos Indígenas de Meso y Sudamérica, SAIIC.

...que se tomen muestras de plantas del territorio donde vive un pequeño grupo de indios huaorani - los Tagaeri- que son un pueblo nómada que vive en lo más profundo de la Amazonia Ecuatoriana. Miller propuso volar hasta una aldea Tagaeri en helicópteros militares, con un pequeño batallón de soldados, un grupo de etnobotánicos norteamericanos, un equipo de filmación que grabara el drama, y otros huaorani en calidad de intérpretes. La idea era tomar muestras de plantas que utilizan los Tagaeri con fines medicinales, llevarlas a California para que fueran estudiadas, y realizar un film comercial sobre ello (Rothschild 1996: 42).

La compañía farmacéutica Merck, en Costa Rica, pagó a ese país mil cien millones de dólares por obtener plantas y recursos genéticos; esto podría parecer muy generoso, sin embargo hay que contextualizarlo ya que Merck vendió, en 1991, ocho mil seiscientos millones de dólares, y esto sin tomar en cuenta las licencias que da a otras compañías por sus patentes (McNally Ruth 1996: 221-228). 
Los Institutos Nacionales de Salud de Estados Unidos han estudiado, en las montañas de Esmeraldas, Ecuador, la secreción de la piel de un batracio, Epidedobates policromo, que tiene poder analgésico doscientas veces más potente que la morfina. Este estudio se realizó a raíz de la información que se obtuvo de parte de los curanderos locales, quienes la han utilizado para friccionar las superficies adoloridas de la piel de sus enfermos (McNally Ruth 1996: 221-228).

Las comunidades indígenas, no obstante que son los depositarias de la tradición herbolaria y guardianas de la materia prima, no tienen acceso a muchos productos farmacéuticos derivados de plantas medicinales y elaborados por los países desarrollados, debido a su alto costo o imposible acceso a través de los sistemas de salud oficiales.

La tramposa lucha de las trasnacionales farmacéuticas en torno al barbasco Discoria composita, Hemsley; planta trepadora originaria de Veracruz, Tabasco y Puebla, y de cuyo tallo se extraen las sapogeninas esteroidales para preparar las píldoras anticonceptivas, es un claro ejemplo del potencial económico que representan las plantas medicinales para la acumulación de capital en los países del norte.

En 1939 la industria farmacéutica Marker llega a México y se une a los Laboratorios Hormona, formando la empresa Syntex, S. A. Posteriormente, Syntex se separa de Marker, se regresa a Estados Unidos y logra sintetizar hormonas a partir de la diosgenina. A inicios de 1954, Marker repunta en el mercado mundial por la venta de esteroides a partir del barbasco debido a que bajó los precios en el mercado mundial. Al mismo tiempo el gobierno mexicano crea su propia industria de esteroides denominada Farquinal. Hacia finales de los años cincuenta, entre 80 y $90 \%$ de la producción mundial de hormonas esteroides provenía de México. Entre julio y noviembre de 1955, seis compañías farmacéuticas norteamericanas, encabezadas por Upjohn, la mayor cliente de Syntex, enviaron su protesta a la embajada de Estados Unidos en México y al secretario de economía en México, rechazando totalmente cualquier política que los obligara a comprarle 
a una empresa determinada. A partir de esta presión, el gobierno mexicano inicia el apoyo a Syntex. Este apoyo se dio en dos formas: primera, mediante decretos presidenciales que fijaban tarifas de exportación prohibitivas a los principales productos manufacturados por otras empresas; y segunda, la negativa de otorgar a los competidores de Syntex los permisos forestales necesarios para recolectar y trasportar el barbasco. Estas medidas cambiaron las cosas para Syntex, ya que inició el control de la industria de esteroides sintéticos en México y en otros países (Gomezjara 1980: 293-301).

Para 1962, por falta de apoyo del gobierno mexicano hacia su empresa Farquinal, esta dejó de funcionar, quedando Syntex con todo el mercado nacional e internacional de esteroides sintéticos. El barbasco y los campesinos que lo producían para su venta y beneficio pasaron a último término (Gomezjara 1980: 293-301).

Esta lamentable experiencia, que conllevó el ahorcamiento por parte del mismo gobierno mexicano de una empresa que trabajaba con números negros, nos demuestra la magnitud del reto que los países en desarrollo tienen que enfrentar ante los intereses económicos de las empresas y gobiernos de las naciones hegemónicas.

\section{LA BIOPIRATERÍA DE LOS RECURSOS DE LA MEDICINA INDÍGENA EN EL ESTADO DE CHIAPAS Y LA LUCHA DEL CONSEJO DE MÉDICOS Y PARTERAS INDÍGENAS TRADICIONALES POR DETENERLA}

Hace aproximadamente treinta años llegó al estado de Chiapas un joven etnobiólogo norteamericano llamado Brent Berlin. Era de su interés investigar el conocimiento milenario que los mayas tenían sobre el uso de las plantas medicinales. Este trabajo, aparentemente inocuo y humanitario para muchos legos, y preponderante para algunos «científicos» con una visión corta de las consecuencias de su quehacer, era la semilla que se estaba preparando para un proyecto comercial de productos farmacéuticos de gran envergadura para el país del norte. Durante este periodo pudo recopilar y enviar al herbario de etnobotánica, situado en los laboratorios de etnobiología en Baldwin Pasillo, 
Universidad de Georgia, en Atenas, Georgia, sin ningún problema, 6,570 colecciones que incluyen 1,500 especies y 160 familias botánicas (UGA 1998). Cabe mencionar que una réplica de esta colección se encuentra en el Herbario de El Colegio de la Frontera Sur, en Chiapas, y una parte en el Herbario del IMSS, empero no existe un solo ejemplar en México de las plantas medicinales colectadas por Berlin en Estados Unidos.

Antes de 1998, el Dr. Berlin inicia gestiones ante el gobierno de Estados Unidos para implementar un proyecto de bioprospección de plantas medicinales mayas en coordinación con Ecosur y el laboratorio Génova de Inglaterra. ${ }^{10}$

A inicios de 1998, la Organización de Médicos Indígenas del Estado de Chiapas, A. C. OMIECH, es invitada a participar en el mencionado proyecto, para lo cual le son explicados, en forma general, los objetivos tendientes a la obtención y venta de fármacos; capacitación para el cultivo de plantas medicinales y desarrollo regional a través de las regalías de las patentes obtenidas por la venta de productos medicinales. La objeción de OMIECH fue en el sentido de la poca claridad y/o ausencia de leyes mexicanas en esta materia y, por consiguiente, el posible surgimiento de problemas posteriores; además, se decidió que el Consejo Estatal de Organizaciones de Médicos y Parteras Indígenas Tradicionales de Chiapas, Compitch, participara activamente en estas negociaciones. Ecosur propuso realizar foros y consultas con expertos para que estas cuestiones legales quedaran claras a ambas partes y, hasta entonces, se daría inicio al proyecto, no importando que pasaran tres o cuatro años en esta etapa.

Efectivamente se realizó un foro en este sentido, pero las cuestiones legales no se aclararon para nadie. En marzo de 1998, a petición de Compitch se realizó otra reunión con los encargados del mencionado proyecto. En ésta se le informó a Compitch sobre la elaboración de un borrador de convenio que sería firmado por Ecosur, la Universidad de Georgia y una compañía de investigación biotecnológica de Inglaterra llamada Molecular Nature Limited; asimismo, se formaría un fideicomiso para recabar las regalías de las 
patentes y una asociación civil, Promaya, que incluiría a todas las comunidades y personas que así lo desearan, con la finalidad de que pudieran presentar sus proyectos para ser evaluados por un comité técnico, el cual determinaría el ulterior financiamiento por parte del fideicomiso. Este fideicomiso empezaría a recibir proyectos cuando la venta de productos farmacéuticos descubiertos produjera regalías, lo que pudiera ser un lapso de tiempo largo, o tal vez nunca, si no se descubriera ningún producto útil a partir de las plantas investigadas.

En julio de 1999, nuevamente Compitch invita a los integrantes del proyecto para que informen sobre los avances de actividades. En esta reunión Ecosur informa que ya firmó el convenio con la Universidad de Georgia y el laboratorio de Inglaterra y que estaban en borradores las propuestas para el fideicomiso y Promaya. El objetivo central del convenio Ecosur-Universidad de Georgia- Laboratorio Molecular Nature Limited, era la investigación de sustancias activas derivadas de las plantas medicinales, para producir medicamentos de patente y ser comercializados.

A partir de ese momento Compitch decide iniciar la lucha pública contra el proyecto del ICBG-Maya; en tanto, el doctor Berlin y el director de Ecosur en ese tiempo, Mario González Espinosa, nunca aceptaron la propuesta de Compitch en el sentido de discutir el problema de manera abierta y con la participación de toda la sociedad. Sobre esta posición Rural Advance Fundation International, RAFI, comentó:

Circo Cerrado: Una reunión cara a cara entre los protagonistas parece necesaria y posiblemente constructiva. Todas las partes confirman que el Consejo ha propuesto una reunión de este tipo y que el ICBG declara que una reunión abierta sería «un circo» y que preferirían una discusión privada entre representantes del ICBG y el Consejo - y quizá otras comunidades mayas-. Los encuentros abiertos en un medio controvertido no son nunca divertidos. Existen, sin embargo, tres razones de peso por las cuales el ICBG debería aceptar esta reunión. Primero, porque el ICBG está pidiendo permiso para hacer algo y no deberá predeterminar las condiciones para la negociación. Si el ICBG no está preparado para negociar en términos locales, no es capaz de conducir este proyecto. Segundo, 
una reunión abierta es un evento que ofrece «oportunidades igualitarias» a todas las partes, para presentar sus puntos de vista y demostrar el nivel de apoyo que tienen sus posiciones en Chiapas. Tercero, la alternativa que propone un encuentro que posteriormente podría ser calificado como «a puertas cerradas» o como discusión «secreta» sería perjudicial para todas las partes. De hecho, en una coyuntura de encuentro abierto, sería lo único que podría servir a los intereses de todos (RAFI 1999).

Las acciones que Compitch implementó para que se suspendiera definitivamente este proyecto estuvieron dentro de los márgenes lícitos y legales. Así, envió un oficio a Semarnap, Conabio, Secretaria de Salud, Conacyt, gobernador del estado de Chiapas, Comisión del Medio Ambiente y Recursos Naturales del Senado de la República, Congreso del estado de Chiapas, INE y Ecosur, en el cual argumentó su inconformidad sobre la operación del proyecto; solicitando la suspensión del mismo y proponiendo que Semarnap y el Senado de la República iniciaran foros con especialistas y consultas a la población para que se estructurará un marco legal concensuado en relación con esta materia.

Por otra parte, inició una campaña amplia de información a las comunidades de la región y el estado sobre las características de este tipo de proyectos, con la finalidad de que ellas decidieran libremente si participaban o no. De igual manera, se llevaron a cabo entrevistas informativas con periodistas de algunos medios de difusión nacionales e internacionales, y se invitó, en varias ocasiones, a todo el personal de Ecosur para que, si lo deseaban, obtuvieran más información de Compitch. Cabe señalar aquí que pocos fueron los investigadores de Ecosur que se acercaron por esta información. Por último, Compitch contactó indígenas y organizaciones nacionales y extranjeras que tenían este tipo de problemas o que eran especialistas en la materia. Con base en todas estas acciones, y principalmente en la campaña de prensa, Semarnap interviene y trata de mediar, conformando una mesa de negociación. Se llevaron a cabo reuniones en el Distrito Federal, Ecosur y Ciesas Sureste, sin llegar a acuerdo alguno. La posición de Compitch fue (es) la de realizar una consulta pública amplia en relación con el acceso a los recursos naturales; la 
propuesta de ICBG-Maya siempre fue en el sentido de una consulta a diez comunidades - cinco del proyecto ICBG-Maya y cinco de Compitch.

En la reunión del mes de mayo del año dos mil, en la ciudad de México, a la cual asistieron funcionarios de Semarnap, INE y Profepa, mediante denuncia de Compitch, el doctor Berlin aceptó haber extraído diversas especies de plantas medicinales mexicanas para llevarlas a Estados Unidos, las cuales «fueron seleccionadas para investigación etnobotánica». El etnobiólogo Berlin se justificó aduciendo que el envío de las plantas a la universidad estadounidense de Georgia fue «de buena fe» (Pérez 2002: 23). Compitch exigió a Semarnap que el doctor Berlin presentara un informe pormenorizado de las especies de plantas que se llevó a Estados Unidos y, además, anexara un inventario de los 35 años de investigación de la herbolaria del sureste mexicano. Pidió a Semarnap buscara en sus archivos el permiso otorgado a Berlin para que exportara plantas. El doctor Berlin presentó una lista de diez plantas que se encuentran en la universidad de Georgia, mas no el informe pormenorizado de sus 35 años de saqueo de plantas en Chiapas. Semarnap no encontró en sus archivos permiso alguno otorgado a Berlin para extraer plantas de México. Ante este delito, Semarnap guardó silencio y no tomó cartas en el asunto.

El apoyo a Compitch — en este proceso de discusión — de asesores externos indígenas y no indígenas, institucionales y no institucionales, fue un elemento - no el único-importante para la cancelación del ICBG-Maya. Un mínimo de asesores tenía la posición de «negociar» con el Proyecto. Su lógica era: «si de todas maneras nos van a robar los recursos naturales, pues hay que negociar y sacar lo mejor que podamos». La contra lógica era: «si te dice un ladrón que, quieras o no, te va a robar en tu casa, que mejor negocies la venta de tus propiedades para que no salgas perdiendo tanto, ¿negociarías?». Ante estas dos posiciones se realizó una consulta en las comunidades de Compitch, aceptándose la segunda. Las comunidades dijeron NO negociamos nuestras plantas. Así, Compitch continuó con su lucha. A partir de este momento Compitch y sus asesores fueron 
calificados por el ICBG-Maya y algunos investigadores de Ecosur, o afines, como intransigentes y radicales.

La información a las comunidades sobre el proyecto ICBG-Maya prosiguió de forma más acentuada. Algunos proyectos de Ecosur, que no tenían relación con el del doctor Berlin, se vieron afectados en su relación comunitaria. Los principales investigadores de ese centro percibieron que podía haber repercusiones sobre otros proyectos y presionaron a su director para que cancelara el proyecto del ICBG-Maya.

Por otro lado, y como un penúltimo intento por salvar su proyecto, el gobierno de Estados Unidos envió al responsable de asuntos económicos de su embajada en México a negociar con los asesores de Compitch: intento infructuoso, pues las comunidades ya habían expresado su decisión. El enviado de la embajada argumentó que su gobierno tenía que proteger las investigaciones e intereses de sus empresas a través del sistema de patentes; además, que los países europeos estaban en gran competencia con Estados Unidos por los recursos naturales de los países que los tenían, y que las plantas medicinales servirían para elaborar medicamentos benéficos para toda la humanidad (Compitch 2000).

El 25 de septiembre del año 2000, a través del oficio 126/200, el director de la Unidad Coordinadora de Análisis Económico y Social de Semarnap, Pedro Álvarez Icaza, dio a conocer la decisión oficial de rechazo al permiso de colecta científica que le presentó ICBG-Maya. Con esto, prácticamente, el proyecto quedaba cancelado (Pérez 2000: 41). El 28 de octubre del mismo año, en comunicado del periódico La Jornada (García 2000: 43), Ecosur desiste de proseguir con el proyecto. No obstante, persiste en dirigirse a los asesores «no indígenas» para que tengan «... un cambio de actitud con base en la convicción de que el diálogo constructivo siempre nos podrá llevar a encontrar nuestros puntos de coincidencia, que buscan impulsar el desarrollo de los pueblos indios y los campesinos de México». 
Pero la situación no terminó ahí. El doctor Berlin declaró: «Han ganado la batalla pero no la guerra». Efectivamente, hizo otro intento por continuar con el saqueo. Organiza a los colectores que habían trabajado hasta ese momento en su proyecto. Los anima a conformar una nueva organización que pudiera contrarrestar a Compitch y que sirviera a sus intereses. Asimismo, les elabora el proyecto ICBG-Maya «modificado» o «disfrazado»; el cual presentan a la Secretaría de Pueblos Indios del Gobierno de Chiapas, Sepi, para ser avalado. Sepi no cae en su juego y rechaza el apoyo al proyecto. Compitch se entera de esta situación y da parte al gobierno estatal. Este interviene y convoca a nueva reunión para mediar. Compitch exige la salida del doctor Berlin de Ecosur. Su director lo defiende, argumentando que el descontento de Compitch es «debido a que es gringo». Compitch argumenta que no es por esa condición sino porque ha violado acuerdos y reglamentos de la propia institución donde labora. Esta argumentación de Compitch no fue tomada en cuenta por el director de Ecosur, ya que él era empleado del doctor Berlin en el proyecto ICBGMaya.

\section{CASI EL FINAL...}

Los intentos del doctor Berlin al presentar, a través de un grupo de sus colectores indígenas, un «nuevo» proyecto al gobierno estatal, para ser avalado por éste, fueron infructuosos. Los integrantes de esta nueva organización, formada por Berlin, denominada Organización para la Conservación de la Medicina Indígena Tradicional de Chiapas, Ocomitch, se percataron de estas intenciones y se deslindaron del etnobiólogo norteamericano. Esta organización, actualmente, sigue su camino paralelo y con los mismos objetivos que los de Compitch.

El doctor Berlin prepara la publicación de su Enciclopedia de la Medicina Maya, continuando así las acciones de apropiación de los conocimientos de los médicos indígenas de Chiapas. 
Compitch, al parecer en descanso, prosigue en su proceso de monitorear a los biopiratas, desarrollando y defendiendo su conocimiento y práctica médica. Sobre esta problemática el doctor Javier Lozoya declaró:

Hoy más que nunca surge para nosotros, los países pobres, una alternativa de convertirnos en productores de nuestros propios medicamentos, adecuando a nuestras necesidades de salud los recursos y las tecnologías que nos son propios; revalorando nuestro bagaje herbolario y desarrollándolo a una nueva dimensión científica y tecnológica que sea aplicada en beneficio de la salud de los mexicanos y que no necesariamente siga los consabidos procedimientos que las sociedades ricas quieren imponer a los demás (Lozoya 1982: 4).

Desafortunadamente, el Dr. Javier Lozoya, quien hasta el momento de la declaración anterior jugó un eminente y reconocido papel en la investigación de la herbolaria medicinal en México, en 2009 se convirtió en agente de ventas de la compañía trasnacional mexicana Genomma Lab. Internacional. ${ }^{11}$ 


\section{LECCIONES Y EXPERIENCIAS APRENDIDAS}

El proyecto operativo ICBG-Maya fue el hilo conductor para lanzar una mirada dialéctica por la herbolaria medicinal; para desnudar elementos escondidos o disfrazados bajo un discurso —y un quehacer- aparentemente «aséptico» y «neutro», que expelen políticos disfrazados de científicos.

Desnudamos algunos de los mecanismos económicos, políticos e ideológicos que el poder utiliza para lograr la acumulación de capital a través de un proceso histórico de fetichización de los recursos herbolarios.

En el trascurso de este ensayo también surgieron preguntas que llevan implícitas algunas posibles respuestas: el cuestionamiento sobre la «asepsia» de las ciencias sociales y, principalmente, las biológicas, supuestamente ajenas a toda carga política e ideológica. La ética, el compromiso social, el involucramiento y los intereses de los sujetos que realizan investigación bio-socio-médica. La pregunta, ¿se debe realizar bioprospección de los recursos de la medicina indígena? Y la respuesta: Sí... pero ¿bajo el control de quién(es)?, ¿para beneficio de quién(es) y quién debe determinar la equidad de estos beneficios?

También el proyecto ICBG-Maya fue un buen pretexto para descubrir que la salud de las poblaciones más desprotegidas económica y socialmente no es el fin último de los mecanismos de fetichización de la herbolaria medicinal, sino la obtención de un «producto» para una «buena venta». Y que en este proceso confluyen intereses trasnacionales con características de paradigma dominante sobre la política institucional del gobierno mexicano; incidiendo y coincidiendo, a la vez, con los intereses de empresas comerciales privadas e instituciones de investigación sociomédica y biológica a escalas nacional y trasnacional.

Una de las lecciones aprendidas por todos los participantes en este proceso, principalmente de los integrantes del ICBG-Maya como de Compitch, es que ningún proyecto de 
bioprospección de herbolaria medicinal a gran escala se puede llevar a cabo sin el consentimiento de la mayoría de la población en donde se va a ejecutar, previa información completa y veraz.

Otra es que los pueblos indígenas, con su participación plena, acuerdos de seguridad y apoyos adecuados, pueden tomar la dirección de proyectos de bioprospección de herbolaria medicinal en sus respectivas regiones.

Por último, demostramos que el proyecto de bioprospección ICBG-Maya, como la mayoría de proyectos de bioprospección en el mundo, tienen no únicamente componentes biológicos, sino también científicos, políticos y sociales escondidos en la fetichización de esta actividad etnobotánica. Que la mayoría esconden, y responden, a intereses propios de naciones que compiten por los recursos naturales de los países pobres, imponiendo políticas y estrategias sobre gobiernos, instituciones, empresas y grupos de poder - económico y político- nacionales. 


\section{REFERENCIAS BIBLIOGRÁFICAS}

Carrera de la Torre, L., 1993, «Gestión Ambiental en el Ecuador. Acuerdo de Contribución entre el Gobierno de Ecuador y el Gobierno de Canadá». En Bioactividad de plantas amazónicas, Víctor Vollacres. Organización de Estados Americanos, Universidad Central de Ecuador, ABYA-YALA, Quito, Ecuador, 1995, p. 12.

Chadler, Melinda, 1993, «The Biodiversity Convention: Select Issues of Interest to International Lawyer». Colorado Journal of International Environmental Law and Policy, v. 4, pp. 141, 174.

Compitch, 2000, «Conversación grabada con el funcionario de la Embajada de Estados Unidos Carl E. Shönander». Encargado del Área Económico Comercial, de la Embajada de Estados Unidos en México, Cinta de sonido única (duración 40 min.), Archivo sonoro Compitch, junio. Para consulta en: compitch@gmail.com.

De la Cruz, Martín, 1991, Libellus de Medicinalibus Indorum Herbis. Fonde de Cultura Económica/Instituto Mexicano del Seguro Social, México.

Downes, David R., 1994, «New Diplomacy for the Biodiversity Trade: Biodiversity, Biotechnology and Intellectual Property in the Convention on Biological Diversity». Touro Journal of Transnational Law, v. 4, pp. 1, 6.

Evans Schultes, Richard, 1993, Plantas de los dioses. Fondo de Cultura Económica, México.

García Barrios, E. Luis y Mario González Espinosa, 2000, «Ecosur y el proyecto de bioprospección ICBG-MAYA en Chiapas». La Jornada, p. 43, 28 de octubre. México. 
Gomezjara, Francisco, 1980, «Fábricas y Supermercados de Salud». En Medicina ¿para quién?, Mercado F. et al., pp. 293-301. Ed. Nueva Sociología, México.

López Medel, Tomás, 1784, Tratado de los tres elementos: agua, aire y tierra, f 187. Biblioteca de la Real Academia de Historia, Madrid, España. En http://repositorioacademico.icesi.edu.co/academico/bitstream/item/2623/1/5.\%20TERCER A\%20PARTE\%20DEL\%20PRESENTE\%20TRATADO,\%20INTITULADO\%20DE\%20L OS\%20TRES\%20ELEMENTOS,\%20EN\%20EL\%20QUAL\%20SE\%20TRATA\%20DEL \%20ELEMENTO\%20DE\%20LA\%20TIERRA.doc [consulta: noviembre de 2002].

Lozoya, Javier, 1997, «Bibliografía Básica sobre Herbolaria Medicinal de México (1984)». En Flora Medicinal de Veracruz, Cano Asseleih, Leticia M., pp. 15-16. Universidad Veracruzana, Xalapa, Veracruz.

Lozoya, Javier y Mariana Lozoya, 1982, Flora Medicinal de México. Instituto Mexicano del Seguro Social, México, D. F.

Massieu Trigo, Yolanda Cristina, 1997, Biotecnología y empleo en la floricultura mexicana. UAM-Azcapotzalco, México.

McNally Ruth and P. Wheale, 1996, «Biopatenting and Biodiversity. Comparative advantages in the new global order». The Ecologist, v. 26, n. 5, pp. 221-228.

Mossinghoff, Gerlad, 1996, «Defending Patents». Letter to- the Editor. The Economist, 612 July, p. 8.

Nadal, Alejandro, 1999, «UNAM/Diversa: el saqueón de los recursos genéticos». La Jornada, 28 de septiembre. México. 
1999a, «UNAM/Diversa: convenio fuera de la ley». La Jornada, 4 de octubre. México.

1999b, «UNAM/Diversa: disfraz de transferencia tecnológica». La Jornada, 11 de octubre. México.

1999c, «Patentes: el acuerdo UNAM/Diversa». La Jornada, 18 de octubre. México.

Naranjo, Plutarco, 1996, «Perspectivas de la Medicina Andina». En Reunión de trabajo sobre políticas de salud y pueblos indígenas, p. 6. Parlamento Andino-Parlamento Indígena de América, 25-27 noviembre, Quito, Ecuador (mimeo).

Nash, Nathaniel C., 1995, «Germany Shuns Biotechnology». The New York Times, pp. D1, 5,4 October.

Organización de Médicos Indígenas del Estado de Chiapas, OMIECH, 1989, «Memorias del cuarto encuentro de OMIECH», p. 72, agosto. San Cristóbal de Las Casas, Chiapas (mimeo).

Pérez Albacete, Martín, 2007, «Introducción al desarrollo y evolución de la Urología en España». Archivos Españoles de. Urología, v. 60, n. 8, p. 1, octubre. Madrid.

Pérez, Matilde, 2000, «Acepta la Universidad de Georgia que extrajo plantas». La Jornada, p. 23, 21 de mayo. México.

2000a, «Negó Semarnap permiso para proyecto de bioprospección ». La Jornada, p. 41, 13 de octubre. México.

Ribeiro, Silvia, 2002, «Medicina Tradicional, patentes y biopiratería». La Jornada, p. 19, 3 de agosto. México. 
Rothschild, David, 1996, Protegiendo lo nuestro. Pueblos indígenas y biodiversidad. Centro por los Derechos Indígenas de Meso y Sudamérica, SAIIC, Quito, Ecuador.

Rural Advance Fundation International, RAFI, Comunicado de Prensa 01/12/99. En http://www.etc.org [consulta: agosto de 2000].

Schultez, R. and F. Raffauf, 1990, «The Healing Forest». Discords Press, v. 2, pp. 9-36. Portland, Oregon.

Sierra, Jorge Luis y Amado Avendaño Villafuerte, 1999, «La cacería de los recursos biológicos». Revista Este Sur, año 6, pp. 10-11, de 11 a 17 de octubre. Tuxtla Gutiérrez, Chiapas.

Torres Galarza, Ramón, 1996, «Producción y venta de plantas medicinales». En Reunión de trabajo sobre políticas de salud y pueblos indígenas, p. 13, 25-27 de noviembre. Parlamento Andino-Parlamento Indígena de América, Quito, Ecuador (mimeo).

Tribble, Jack L., 1995, «Gene Ownership versus Access: Meeting the Needs». National Agricultural Biotechnology Council Reports, pp. 97-104. Washington, D. C.

UGA, «Laboratories of Ethnobiology. Resources.Ethnobotany Database». En http:// http://obberlin.myweb.uga.edu/resources.html [consulta: diciembre de 1998]. 


\section{Notas}

${ }^{1}$ Cada año, desde 1994 y durante dos semanas, se reúne la Conferencia de las Partes, CDP. Cerca de mil individuos asisten a las CDP, la mitad son funcionarios oficiales de los países y la otra mitad representantes de ONG más unos cuantos académicos.

${ }^{2}$ El preámbulo de CDB menciona: «Las Partes Contratantes: Reafirmando que los Estados tienen derechos soberanos sobre sus propios recursos biológicos...». Al principio del art. 3: «...los Estados tienen el derecho soberano de explotar sus propios recursos en aplicación de su propia política ambiental...».

${ }^{3}$ La Tercera Reunión de la Mesa de Negociación Tripartita no se llevó a cabo debido a que únicamente se presentó Compitch. Estaría conformada, en San Cristóbal de Las Casas, para la discusión sobre el proyecto ICBG-Maya e integrada por: El Colegio de la Frontera Sur, ICBG- Maya, Semarnap y Compitch; se efectuaría en el mes de noviembre del año 2000.

${ }^{4}$ El documento mimeografiado de los pronunciamientos y declaraciones del Consejo de Organizaciones de Médicos y Parteras Indígenas Tradicionales de Chiapas (COMPITCH), en esta mesa, se pueden solicitar al correo electrónico: compitch@gmail.com, o ver en: Castro Soto, Juan, 2000, «Pukuj. Biopiratería en Chiapas», p. 52, agosto. REMALC/CIEPAC/COMPITCH, San Cristóbal de Las Casas, Chiapas.

${ }^{5}$ Información sobre los proyectos: ICBG-Maya (México); ICBG-Zonas Áridas (México); ICBG-Bioactive Agents from Dry land Plants in Latin America (Chile y Argentina), en la pág. web:http://www.ag.arizona.edu/OALS/ICBG/publications/noticiero5-2 /Timmermann [consulta: julio de 1997).

${ }^{6}$ Alfonso Pérez Grovas, coordinador del sistema SEP-Conacyt, mencionó ante la comisión de Ciencia y Tecnología de la Cámara de Diputados que con el recorte presupuestal para el año 2000 «no podremos pagar ni el teléfono ni la luz», además «...se pone en riesgo la existencia de centros de investigación de alto nivel de eficiencia que cuentan con 2,000 científicos, de los cuales la mitad tiene grado de doctor... y que atienden 10,000 empresas». La Jornada, 20 octubre de 1999.

${ }^{7}$ «Convenio para la protección de Derechos de Propiedad Intelectual y Distribución de Beneficios del ICBGMaya». Convenio que regula la protección de la propiedad intelectual y la distribución de beneficios del proyecto «Investigación farmacéutica y uso sustentable del conocimiento etnobotánico y biodiversidad en la región maya de Los Altos de Chiapas». UGARF, ECOSUR y MNL son partes de este convenio; San Cristóbal de Las Casas, Chiapas, 1999; Memorándum interno de ECOSUR (mimeo).

${ }^{8}$ Una copia del expediente técnico del proyecto del International Collaborative Biodiversity Group (ICBGMaya) denominado «Drug Discovery and Biodiversity Among the Maya of Chiapas» se puede consultar en las oficinas de la Organización de Médicos Indígenas del Estado de Chiapas, A. C. (OMIECH), correo electrónico: omiech@ prodigy,net.mx

9 En el expediente técnico del proy. del ICBG-Maya, pp. 8, 40, 74, 141, 203 (copia del original, proporcionada por el Dr. Brent Berlin) «Drug Discovery and Biodiversity Among the Maya of Mexico», podemos analizar las partidas presupuestales correspondientes al personal adscrito a este proyecto de bioprospección - el presupuesto no incluye los salarios base que los participantes reciben en la institución donde laboran normalmente, como Ecosur- La secretaria del líder del proyecto recibe un sobresueldo de 10,000 pesos mensuales durante el primer año. Los puestos especializados — biólogos, bioquímicos — reciben 25,800 pesos mensuales de sobresueldo.

${ }^{10}$ Comunicación del economista ambiental José Carlos Fernández Ugalde, de Ecosur, a la mesa directiva de la Organización Médicos Indígenas del Estado de Chiapas (reunión de enero de 1998). Oficio enviado a OMIECH, por parte del colaborador de Ecosur, en proyecto ICBG-Maya, José Carlos Fernández Ugalde, (agosto de 1999). Se localiza en el Archivo de Compitch, San Cristóbal de Las Casas, Chiapas. Correo electrónico: compitch@gmail.com

${ }^{11}$ La participación del Dr. Lozoya en los Laboratorios Genomma Lab Internacional se puede ubicar en la dirección electrónica: http://www.expansion.com.mx/nivel2.asp?cve=1002_20\&xsl=print.xsl\&y=1 y en http://www.genommalab.com/Cidet.php [consulta: 26 de noviembre de 2009]. 


\section{CONCEPTOS}

La Biodiversidad o diversidad biológica comprende toda variedad de seres vivos que existen en la tierra, en el mar o en los ríos.

Los seres vivos son la materia prima a partir de la cual se pueden obtener recursos genéticos.

Los Recursos Genéticos son muestras o extractos de material orgánico, tales como plantas, hongos, insectos, animales e incluso genes humanos, que tienen un uso potencial o real para la humanidad.

Partiendo de los conceptos anteriores definiremos a la Bioprospección como la exploración, extracción e investigación de la diversidad biológica y los conocimientos indígenas en busca de recursos genéticos y bioquímicos de valor comercial (Rothschild 1996:35).

Siguiendo este último concepto, la Biopiratería es la apropiación o patentización de recursos genéticos y bioquímicos, provenientes del conocimiento indígena, principalmente, por parte de países del Hemisferio Norte, sin reconocimiento o compensación justa y proporcional a los beneficios que, en última instancia, se derivarán de los conocimientos originarios.

Una de las características constantes de esta apropiación, por parte de los países ricos, es la falta de Consentimiento Fundamentado Previo que se define como: el consentimiento para llevar a cabo una actividad, otorgado después de recibir información completa sobre las razones para llevar a cabo dicha actividad, los procedimientos específicos que entraña, los riesgos potenciales y todas las implicaciones que puedan ser previstas de manera realista (Rothschild 1996: 37). 
Fecha de recepción 22 de noviembre de 2009

Fecha de aceptación: 9 de agosto de 2010 